haps this will be added to their number. Professor Flygare clearly hopes for more-for example, by challenging the reader with problems at the end of each chapter.

The difficulty with an epic work is that one dare not be discursive or one cannot get it all between two hard covers. Let me take, for example, the section on scattering in this book. The fifty pages devoted to this topic is sufficient to include topics such as vibrational and rotational inelastic scattering or the relationship between the potential energy surface for a reaction and the energy distribution in the products.

\section{Mitochondrial and plastid heredity}

Organelle Heredity. By N. W. Gillham. Pp. 602. (Raven: New York, 1978.) $\$ 64.35$.

Non-MEndelian Heredity has been the Cinderella of genetics ever since Correns discovered it exactly 70 years ago. It has long been complicated, confused and experimentally intractable. It is still complicated, but powerful new techniques, ably described in this new book by one of their chief wielders, are rapidly reducing the confusion.

For over a decade it has been clear that there are two fundamentally different causes of non-Mendelian heredity. One of these, the direct inheritance of preformed cell structure-seemingly independently of DNA - is well established only for the ciliate cortex. The more widespread cause is the existence of DNA inherited other than in a Mendelian fashion. Sometimes such DNA is nuclear-B-chromosomes are the most obvious example-but more usually it is cytoplasmic. Although there are numerous scattered cases where cytoplasmic DNA constituting the genome of intracellular viruses or cellular endosymbionts causes nonMendelian inheritance, cytoplasmic DNA occurs regularly only in plastids and mitochondria; the genetics of these, not of organelles in general, is the subject of Gillham's excellent hook.

The book is the most thorough treatment yet of mitochondrial and plastid genetics, and deserves to be read widely by geneticists, biochemists, cell biologists and advanced students. Although it excludes evolutionary discussions, it will be invaluable to all interested in organelle evolution. Fifteen of the twenty-one chapters concentrate on transmission genetics, the four on baker's yeast and the four on Chlamydomonas forming the heart of the book.
There is no mention of the Born approximation or of semi-classical quantisation (apart from the Bohr atom). The book would not be sufficient in itself for a postgraduate course on molecular dynamics and would be a little thin even for an advanced undergraduate course on this topic.

Perhaps I am being petty. It is in the main a fine work which I shall find very useful. I doubt, however, that it will be adopted as a course book in many British universities.

J. N. Murrell

J. N. Murrell is Professor of Chemistry at the University of Sussex, Brighton, UK.

Nuclear transmission genetics was substantially worked out within a single decade of Morgan's first concentration on Drosophila. Yet although Ephrussi and Sager made yeast and Chlamydomonas the drosophilas of cytoplasmic heredity decades ago, we still do not understand it. This is because until the past decade the characteristic uniparental inheritance of organelle genes, and the scarcity of different mutants, precluded traditional genetic analysis of segregation and recombination. Much of the recent progress stems from strictly genetic advances-the ability to obtain biparental inheritance and to induce and isolate a greater variety of mutants. This has led to an impressive array, often bewildering to the outsider, of new mapping techniques; and it is valuable to have them so clearly laid out in one place.

The discovery of organelle DNA has also been important, especially on the psychological side as a booster of the intellectual respectability of the field. Wherever possible Gillham integrates the molecular and genetic approaches. but where this cannot be done, as in the conflicting data on the inheritance of Chlamydomonas chloroplast DNA, he simply describes the basic data. His account of the conflicts between Sager's work and his own are admirably balanced. Particularly valuable are the comparisons between the yeast and Chlamydomonas work. Although yeast is simpler and more intensively studied, in the long run Chlamydomonas will be ideal for studying the interactions between biparentally inherited nuclear DNA, 'maternally' inherited plastid DNA and its 'paternally' inherited mitochondrial DNA. ('Maternal' and 'paternal' are peculiarly inappropriate for Chlamydomonas which is isogamous and has an organelle like the acrosomal tubule of sperm on its so-called 'maternal' gametes.)

Mitotic segregation prevents enumeration by the cis-trans test of the polypeptides coded by organelle DNA. but genetic techniques can help in assigning specific gene products to nuclear or organelle DNA: Gillham ably describes the combined genetic and biochemical study of mutants, the use of interspecific hybridisation and the analysis of mammalian cell hybrids in tissue culture. DNA hybridisation studies of mules and hinnies have shown mitochondrial DNA to be maternally inherited, as in fungi. Here, however, the mechanism may simply be dilution of sperm mitochondria by the vast excess of female ones in the egg. In fungi, many of which are isogamous like Chlamydomonas, this cannot be so; curiously, twelve years after I discovered chloroplast fusion in Chlamydomonas the cytological fate of mitochondria in fungal zygotes is still unknown, although in 1978 Gaffal demonstrated mitochondrial fusion in zyotes of the alga Polytoma. As Gillham points out, cytology is lagging behind genetics and biochemistry.

Although emphasising genetics, Gillham also summarises present biochemical, cytological and physiological knowledge of the structure and functions of organelle DNA. The first chapter is an up-to-date outline of the structure and function of plastids and mitochondria. It is followed by one on the structure and replication of mitochondrial and chloroplast DNA, including brief accounts of the underlying techniques. The two chapters on the RNA- and protein-synthetic machinery of plastids and mitochondria, and their biogenesis, describe the evidence that organelle RNA is coded by organelle DNA; the majority of proteins, on the other hand, including nearly all the ribosomal ones, are coded in the nucleus and synthesised outside the organelles.

The remaining two chapters discuss the biosynthesis of the mitochondrial inner membrane and the thylakoids. Here also most of the proteins are coded by the nuclear DNA; only a handful are yet known to be coded by the organellar plasmids. I suspect that the bulk of this DNA-like nuclear DNA-does not code for proteins. The pace of advance of both the genetic and the biochemical methods Gillham so clearly describes is such that we shall soon know; things too recent to be included are constantly being discovered, like the presence of split genes in both mitochondria and plastids.

This well produced book, with its ample references, is a milestone in the history of genetics.

T. Cavalier-Smith

T. Cavalier-Smith is Lecturer in Biophysics at King's College. University of London, $U K$. 